\title{
Dispersion of helically corrugated waveguides: Analytical, numerical, and experimental study
}

\author{
G. Burt, ${ }^{1}$ S. V. Samsonov, ${ }^{2}$ K. Ronald, ${ }^{1}$ G. G. Denisov, ${ }^{2}$ A. R. Young, ${ }^{1}$ V. L. Bratman, ${ }^{2}$ A. D. R. Phelps, ${ }^{1}$ A. W. Cross, ${ }^{1}$ \\ I. V. Konoplev, ${ }^{1}$ W. He, ${ }^{1}$ J. Thomson, ${ }^{1}$ and C. G. Whyte ${ }^{1}$ \\ ${ }^{1}$ Department of Physics, University of Strathclyde, Glasgow, G4 ONG, Scotland, United Kingdom \\ ${ }^{2}$ Institute of Applied Physics, Russian Academy of Sciences, Nizhny Novgorod, 603950, Russia
}

(Received 14 April 2004; published 7 October 2004)

\begin{abstract}
Helically corrugated waveguides have recently been studied for use in various applications such as interaction regions in gyrotron traveling-wave tubes and gyrotron backward-wave oscillators and as a dispersive medium for passive microwave pulse compression. The paper presents a summary of various methods that can be used for analysis of the wave dispersion of such waveguides. The results obtained from an analytical approach, simulations with the three-dimensional numerical code MAGIC, and cold microwave measurements are analyzed and compared.
\end{abstract}

DOI: 10.1103/PhysRevE.70.046402

PACS number(s): 52.70.- m, 41.60.Cr, 84.40.Ik, 41.20.Jb

\section{INTRODUCTION}

Metal hollow waveguides with various types of periodic corrugation are widely used in high-power microwave electronics. One such structure that has recently attracted considerable interest consists of a helical corrugation in the wall of a circular cylindrical waveguide, which involves both axial and azimuthal periodicity. This provides asymmetry of the wave dispersion for circularly polarized modes, resulting in additional mode selection. These properties make waveguides with a helical corrugation attractive for a large number of applications. In particular, they have been used as slow-wave interaction structures in relativistic Cherenkov devices [1], in Bragg reflectors [2], and as mode converters (see, e.g., [3]), etc. Helically corrugated waveguides have recently been successfully used as interaction regions in gyrotron traveling-wave tubes (GTWTs) [4,5], and gyrotron backward-wave oscillators (GBWOs) [5-7] and as a dispersive medium for passive microwave pulse compression [8]. Due to this wide applicability, it is relevant and important to investigate the electrodynamic properties of such waveguides by analytical and numerical techniques, confirming the validity of the methods by comparison with experimental measurement.

The most general and simple method for theoretical analysis of the dispersion characteristics and electromagnetic field structure in waveguides with any type of shallow corrugation is the method of perturbation, which is based upon replacement of the corrugated surface by a cylindrical surface but with fictitious magnetic currents $[9,10]$. This method provides reasonable accuracy when the corrugation depth is much smaller than the operating wavelength. For the theoretical analysis of waveguides with comparatively large amplitude helical corrugations, it is possible to use one of the available computer codes based on the finite-difference evaluation of the full three-dimensional (3D) Maxwell equations. In this paper the time-domain code MAGIC [11] is used to simulate wave propagation through the structures, with the results from the simulations compared to those from the method of perturbation and experiments. Two different experimental methods of measuring the wave dispersion are also presented in this paper.
The waveguides studied were used in GTWT/GBWO experiments [4,7] and in frequency-swept pulse compression experiments [8]. It is important to note that these applications rely on significantly different mode dispersion for their operation. For a GTWT the most favorable operating wave is that which has a constant and sufficiently high group velocity over a wide frequency band in the region of close-to-zero axial wave number. In contrast, the operating wave for a pulse compressor should have a strong frequency dependent group velocity over a frequency band which is separated from the cutoff frequency of the waveguide. These very different requirements can both be satisfied by waveguides with quite similar geometry. In both cases the parameters of the helical corrugation are chosen such that two modes, one close to cutoff and one propagating, are resonantly coupled.

In Sec. II the principles of synthesizing the necessary dispersion and its qualitative characteristics are discussed. In Sec. III an analytical approach is presented which is based on solution of a dispersion equation derived from the coupledmode theory. In Secs. IV and V the techniques used to obtain the wave dispersion from experimental measurement and from MAGIC simulations, respectively, are presented. In Secs. VI some examples of helically corrugated waveguide are analyzed, and results obtained from the various methods presented are compared and discussed.

\section{PRINCIPLE OF THE DISPERSION SYNTHESIS}

Let us consider a waveguide with the helical profile of its inner surface represented in a cylindrical coordinate system $(r, \varphi, z)$ as follows:

$$
r(\varphi, z)=r_{0}+l \cos \left(m_{B} \varphi+k_{B} z\right) .
$$

Here $r_{0}$ is the mean radius of the waveguide, $l$ is the amplitude of the corrugation, $m_{B}$ and $k_{B}=2 \pi / d$ define the azimuthal number and axial component of the Bragg periodicity vector, respectively, and $d$ is the corrugation period. For simplicity, consider only two circularly polarized modes 1 and 2 having azimuthal numbers $m_{1}$ and $m_{2}$, respectively (the positive value of $m$ is defined for right-handed modes), with axial wave numbers $k_{z 1}$ and $k_{z 2}$ in the absence of the corrugation 


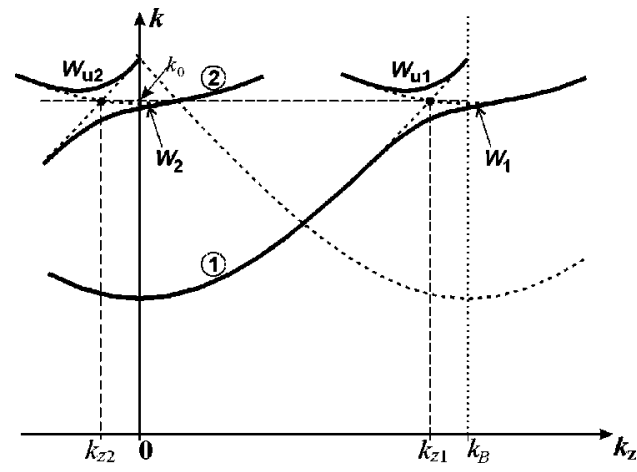

FIG. 1. Schematic dispersion diagram for a helically corrugated waveguide.

$(l=0)$ obeying the dispersion equation for a smooth waveguide:

$$
k^{2}=k_{z i}^{2}+k_{t i}^{2}, \quad i=1,2,
$$

where $k=\omega / c$ is the wave vector ( $\omega$ is the angular frequency, $c$ is the speed of light), $k_{t i}=\nu_{i} / r_{0}$ is its transverse component for each mode, and $\nu_{i}$ is the root of the derivative of the corresponding Bessel function (here $\nu_{2}>\nu_{1}$ ). In a periodically corrugated waveguide the electromagnetic field can be represented as a superposition of the spatial harmonics, which, at negligibly small corrugation amplitude, possess the dispersion characteristics of the smooth waveguide modes [Eq. (2)] (partial modes) shifted along the axial-wavenumber axis by an integer number of the Bragg periodicity vector $k_{B}$ (Fig. 1). At nonzero amplitude of the corrugation, resonant coupling between the modes occurs when their axial and azimuthal wave numbers satisfy the Bragg conditions

$$
k_{z 1}-k_{z 2}=k_{B}, \quad m_{1}-m_{2}=m_{B} .
$$

This coupling results in the appearance of eigenwaves whose dispersions can be represented as splitting of the partial mode dispersions near the frequencies of their intersections with dispersions of the spatial harmonics as shown in Fig. 1.

A helical wall perturbation can provide selective coupling between a higher and a lower circularly polarized mode, avoiding the Bragg reflection zones, which would inevitably appear in the case of an axisymmetric corrugation because of coupling between, for example, forward- and backwardpropagating spatial harmonics of mode 1. If the coupling occurs in the frequency region close to the cutoff of mode 2 , $\left|k_{z 2}\right| \ll k_{0}$ (here and henceforth we will use the designation of $k_{z 1}$ and $k_{z 2}$ for the values of the corresponding axial wave numbers taken at the wave vector $k_{0}$ of exact Bragg resonance), then one of the eigenwaves, namely, the fundamental spatial harmonic (mode $W_{2}$ in Fig. 1), which can be considered as a modified mode 2, can have a dispersion that is attractive for either gyro devices or pulse compressors. This eigenwave can have a positive group velocity at zero axial wave number.

In fact, a larger corrugation amplitude (which controls the dispersion splitting) and a zero or positive value of $k_{z 2}$ (which is controlled by the corrugation period) result in a

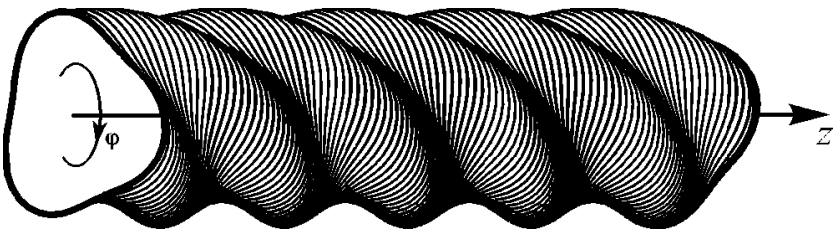

FIG. 2. Schematic view of a waveguide with a threefold righthanded helical corrugation.

larger eigenwave group velocity, which can be made almost constant over a wide frequency band. Moreover, the cutoff frequency of the undesirable upper eigenmode $W_{u 2}$ (Fig. 1) can be sufficiently upshifted. In this case, the mode $W_{2}$ is attractive as an operating mode in a GTWT. In contrast, if the coupling is sufficiently weak and the value of $k_{z 2}$ is negative then the group velocity of the eigenwave changes from a high value of the mode 1 to a very small value of mode 2 within a rather narrow frequency band. With an appropriate choice of parameters, it is possible to avoid regions with zero or negative group velocity, and then this configuration becomes favorable for the realization of pulse compression.

In both cases the frequency band of main interest is mostly situated below the cutoff frequency of mode 2 , and therefore, by having a sufficiently smooth down-tapering of the corrugation amplitude, the eigenmode, which is essentially a superposition of spatial harmonics $W_{2}$ and $W_{1}$, totally transforms into partial mode 1 . In practice, an operating structure consists of a section with a regular corrugation bounded on each side by a taper with gradually tapered corrugation amplitude that meets a waveguide having mean radius $r_{0}$, with the lower mode 1 used to inject and extract the microwave energy from the structure. Therefore, in this paper, the operating eigenwave of a helically corrugated waveguide will be interpreted as the modified or strongly perturbed mode 1 , and will be represented in the dispersion by the mode $W_{1}$ shown in the diagrams. In experiments with the use of the helical waveguides in gyro devices and in pulse compression experiments a threefold $\left(\left|m_{B}\right|=3\right)$ helical corrugation (Fig. 2) was used that coupled the $\mathrm{TE}_{2,1}$ (mode 2) and the $\mathrm{TE}_{1,1}$ (mode 1 ) circularly polarized modes of opposite rotation.

\section{ANALYTICAL APPROACH}

When the corrugation amplitude $l$ is small compared with the wavelength, the modes of the helically corrugated waveguide can be found using the method of perturbation and the coupled-mode theory $[10,12]$, leading to the eigenmode dispersion equation

$$
\left(k^{2}-k_{z}^{2}-k_{t 1}^{2}\right)\left[k^{2}-\left(k_{z}-k_{B}\right)^{2}-k_{t 2}^{2}\right]=4 \kappa^{2} k_{0}^{4},
$$

where

$$
\kappa=\frac{l}{2 r_{0}^{3} k_{0}^{2}} \frac{\nu_{1}^{2} \nu_{2}^{2}-m_{1} m_{2} r_{0}^{2}\left(k_{0}^{2}+k_{z 1} k_{z 2}\right)}{\sqrt{\left(\nu_{1}^{2}-m_{1}^{2}\right)\left(\nu_{2}^{2}-m_{2}^{2}\right)}}
$$

is the coupling coefficient normalized to the value of the wave vector $k_{0}$ of exact Bragg resonance. If the coupling 
coefficient is negligibly small, Eq. (4) splits into two equations describing the dispersion of the uncoupled mode 1 and the first Floquet harmonic of mode 2. When the coupling is small, such that $\kappa \ll 1$, and the Bragg conditions (3) are satisfied exactly at the cutoff frequency of mode $2\left(k_{0}=k_{t 2}, k_{z 2}\right.$ $=0$ ), the eigenwave dispersions are split in the wave vector by $\pm \kappa k_{0}$ at $k_{z}=k_{B}$. Further analysis will be concentrated on properties of the wave $W_{1}$ (Fig. 1) whose perturbation theory dispersion $k\left(k_{z}\right)$ or $f\left(k_{z}\right)(f=k c / 2 \pi$ is the frequency) [Figs. 5(a), 6(a), and 7(a) represents a numerically found selected root of Eq. (4) plotted with sufficiently small intervals over the $k_{z}$ axis. In this case, the eigenwave group velocity shown in the diagrams [Figs. 5(b), 6(b), and 7(b)] is numerically derived using the following expression:

$$
v_{g r}=c \frac{d k}{d k_{z}} .
$$

\section{EXPERIMENTAL METHODS OF DISPERSION MEASUREMENT}

Two different techniques were used to measure the dispersion of the eigenwave of a helically corrugated waveguide. The first method, which relies on the asymmetry of propagation through the structure of the two circularly polarized waves, was first applied to helical waveguide dispersion measurement in Ref. [13]. In the experiments discussed here the structures had a threefold right-handed helical corrugation (Fig. 2), $m_{B}=-3$, which in the lowest-frequency region resonantly coupled a forward-propagating left-handed $\mathrm{TE}_{-1,1}$ mode $\left(m_{1}=-1\right)$ and a near-cutoff right-handed $\mathrm{TE}_{2,1}$ mode $\left(m_{2}=2\right)$. In this case, when the $\mathrm{TE}_{-1,1}$ wave is introduced through a taper with a gradual increase in the corrugation amplitude it is transformed into the eigenmode $W_{1}$, having a significantly modified dispersion (designated as $k_{z}^{-}$), whereas the right-handed $\mathrm{TE}_{1,1}$ wave remains practically unperturbed $\left(k_{z}^{+} \approx k_{z \mathrm{TE} 1,1}\right)$. The difference in axial wave numbers of two counterrotating components $\Delta k_{z}=k_{z}^{-}-k_{z}^{+}$forming a linearly polarized $\mathrm{TE}_{1,1}$ mode results in the rotation of its polarization vector by the angle $\Delta \varphi=\Delta k_{z} L / 2$, where $L$ is the effective length of the helical structure (this effect is well known for gyrotropic media in optics).

In the experiments, the input radiation was launched as a fundamental mode of a standard rectangular waveguide and then transformed into the linearly polarized $\mathrm{TE}_{1,1}$ mode by passing through a rectangular-to-circular adapter. This mode consecutively passed a helical up-taper (three and four periods long with linear increase in the corrugation amplitude), a long section with a constant corrugation amplitude, and a helical down-taper to a circular waveguide. A receiver detecting linearly polarized radiation was placed on the axis of the helical structure and was separated in space to reduce the reflections. A scalar network analyzer (SNA) was used to measure the transmittance of the system as a function of frequency. It is clear from a qualitative analysis that the difference in the axial wave numbers $\Delta k_{z}$ and, hence, the polarization turn $\Delta \varphi$ is an increasing function of the frequency; therefore, when using a sufficiently long helical structure and
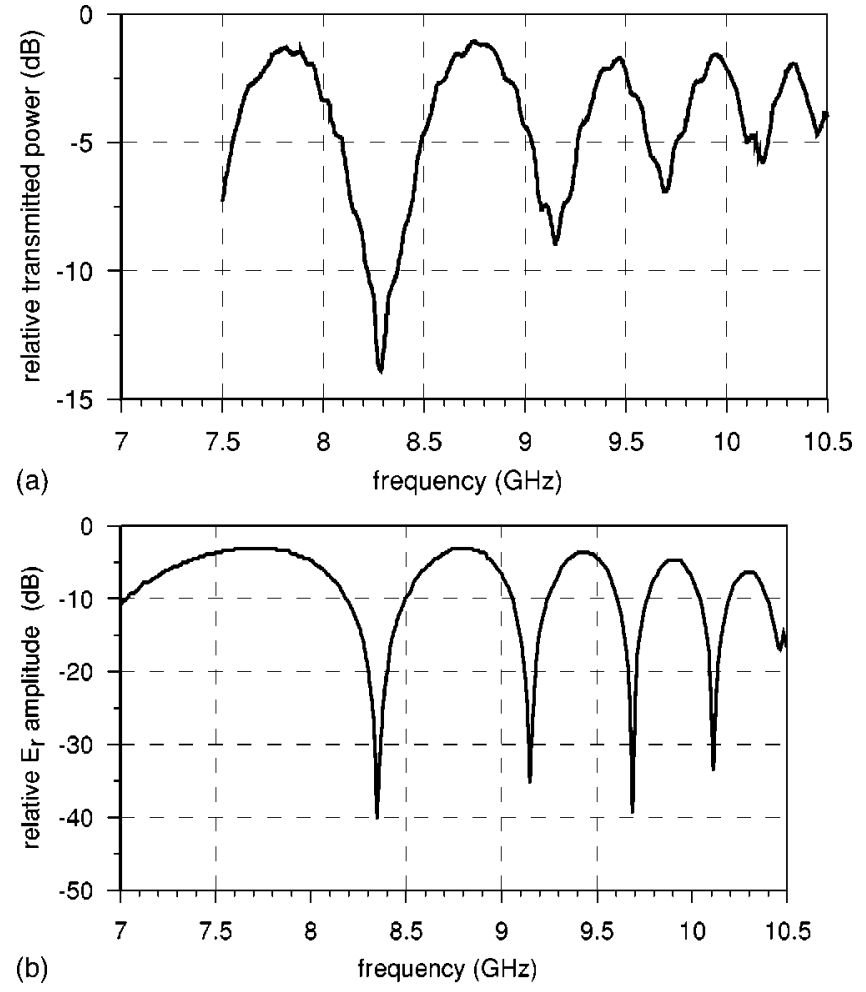

FIG. 3. Experimental and numerical results for the GTWT waveguide: (a) SNA measurements; (b) equivalent SNA MAGIC simulations.

a fixed orientation of the receiving port, a SNA frequency scan represented a number of minima (almost zeros) and maxima [Fig. 3(a)] which corresponded to polarizations of the output radiation perpendicular or parallel to the polarization of the receiving port. The frequencies of the minima, $f_{n}$, sharply indicated on a logarithmically scaled SNA plot [Fig. 3(a)], were accurately measured and the points for the dispersion diagram were calculated as follows:

$$
k_{z}^{-}\left(f_{n}\right)=k_{z \operatorname{TE} 1,1}\left(f_{n}\right)+\frac{2}{L}\left(\frac{\pi}{2}(2 n-1)+\varphi_{R}\right), n=1,2, \ldots,
$$

where $\varphi_{R}$ is the angle of the azimuthal orientation of the rectangular waveguide in the receiving port with respect to that in the launching port. It was assumed that the two helical tapers of length $L_{\text {tap }}$ each acted together like a single piece of waveguide with a regular corrugation of length $L_{t a p}$, i.e., in Eq. (7) $L=L_{r e g}+L_{\text {tap }}$, where $L_{\text {reg }}$ is the length of the section with regular corrugation. By varying the angle $\varphi_{R}$ within an interval of $\pi / 2$, a sufficient number of points for the dispersion diagram can be measured (in the measurements of long structures for the pulse compressor only one orientation was needed, while for a shorter structure of the GTWT two angles $\varphi_{R}=0$ and $\varphi_{R}=\pi / 2$ were used).

When the experimental equipment did not allow measurements of the minimal frequency $f_{1}$, the uncertainty in the number of $180^{\circ}$ polarization turns [integer $n$ in Eq. (7)] was discovered by measuring structures of different lengths. The points of the dispersion characteristic found by this method 

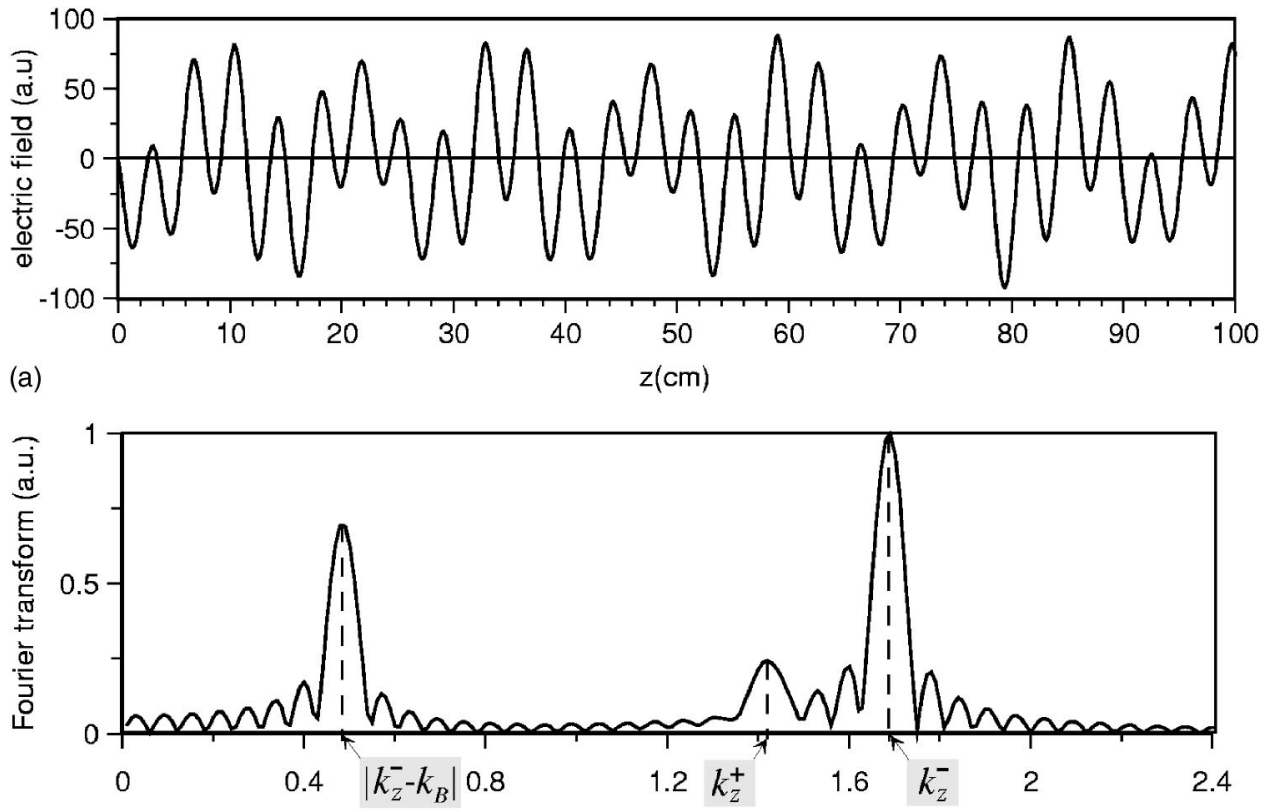

(b)

were interpolated by a polynomial which was used to derive the group velocity as a smooth function of the frequency in accordance with Eq. (6). The order of the polynomial was increased until further increments did not significantly influence the function that was obtained (fourth and fifth order polynomials were used in Figs. 5-8 below).

The experimental approach discussed relies on the approximation that the right-handed (weakly perturbed) wave in the helical structure has the same dispersion relation as the $\mathrm{TE}_{1,1}$ mode of the smooth circular waveguide. However, a large corrugation amplitude can result in a considerable change in the latter dispersion and therefore lead to systematic inaccuracy. Another source of inaccuracy in this method is the inexact estimation of the effective length of the structure as $L=L_{\text {reg }}+L_{\text {tap. }}$ Clearly, this inaccuracy can be diminished by increasing the length of the regular corrugation section with respect to the length of the tapers.

The second experimental method was free from the sources of systematic inaccuracy detailed above. It was based on the use of a vector network analyzer (VNA) to directly and accurately measure the difference between the phases of the signal at the input and output calibration planes as a function of the frequency. In fact, the phase difference $\Delta \psi$ between the eigenwave electromagnetic fields taken at two different cross sections separated by length $D$ can be determined as $\Delta \psi=k_{z} D$. Therefore, by measuring the phase $\Delta \psi$, the eigenwave axial wave number $k_{z}$ can be measured if the experimental setup provides excitation of a sufficiently pure eigenwave in a piece of regularly corrugated waveguide, $D$.

In experiments with helically corrugated structures, the excitation of an eigenwave was ensured by introducing a circularly polarized $\mathrm{TE}_{1,1}$ mode to a regularly corrugated section through the helical taper mentioned above. The circularly polarized $\mathrm{TE}_{1,1}$ mode (right or left handed) was converted from a linearly polarized wave by passing it through a broad-frequency-band polarizer having in the middle an el- liptical cross section azimuthally oriented by $+45^{\circ}$ or $-45^{\circ}$ with respect to the polarization vector of the incident wave. According to the chosen method, the measured structure consisted of a relatively short section of regular corrugation which was bounded on each side by a helical taper, polarizer, circular-to-rectangular adaptor, and waveguide-to-cable

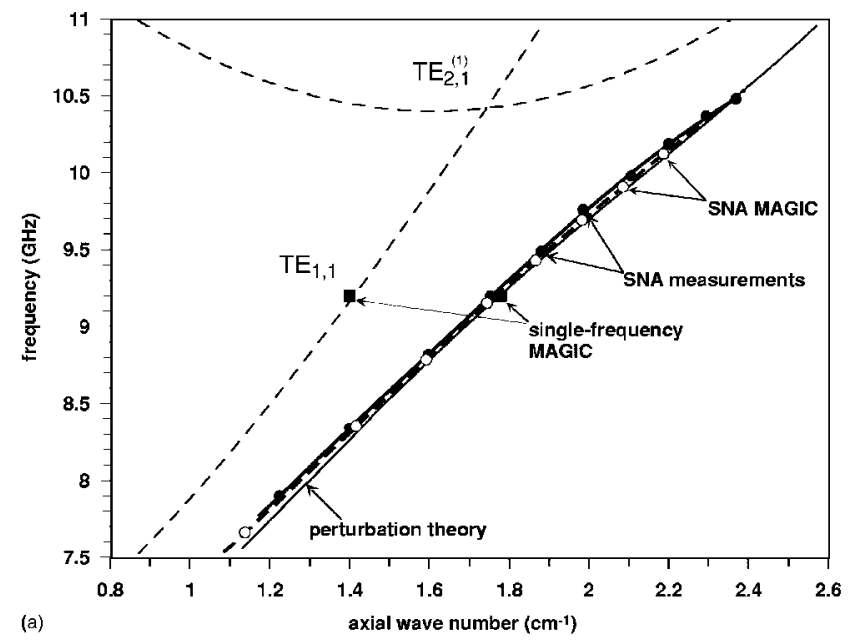

(a)

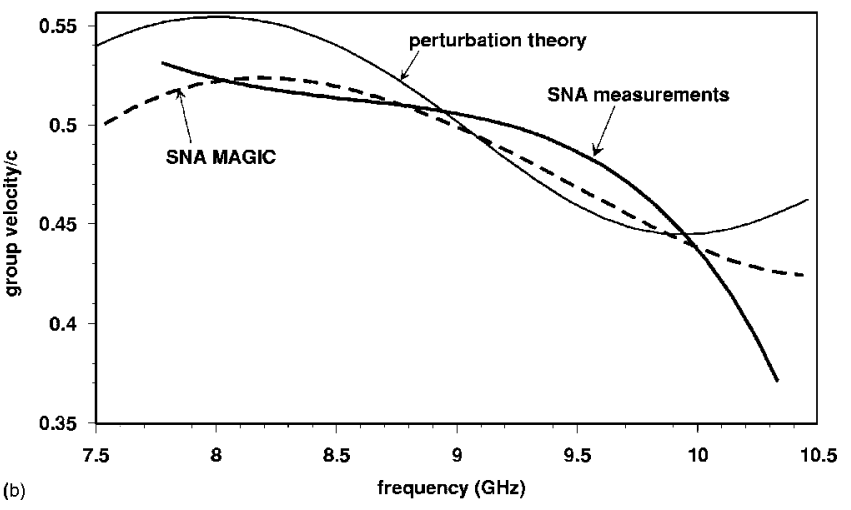

FIG. 5. Dispersion characteristics for the GTWT waveguide. 

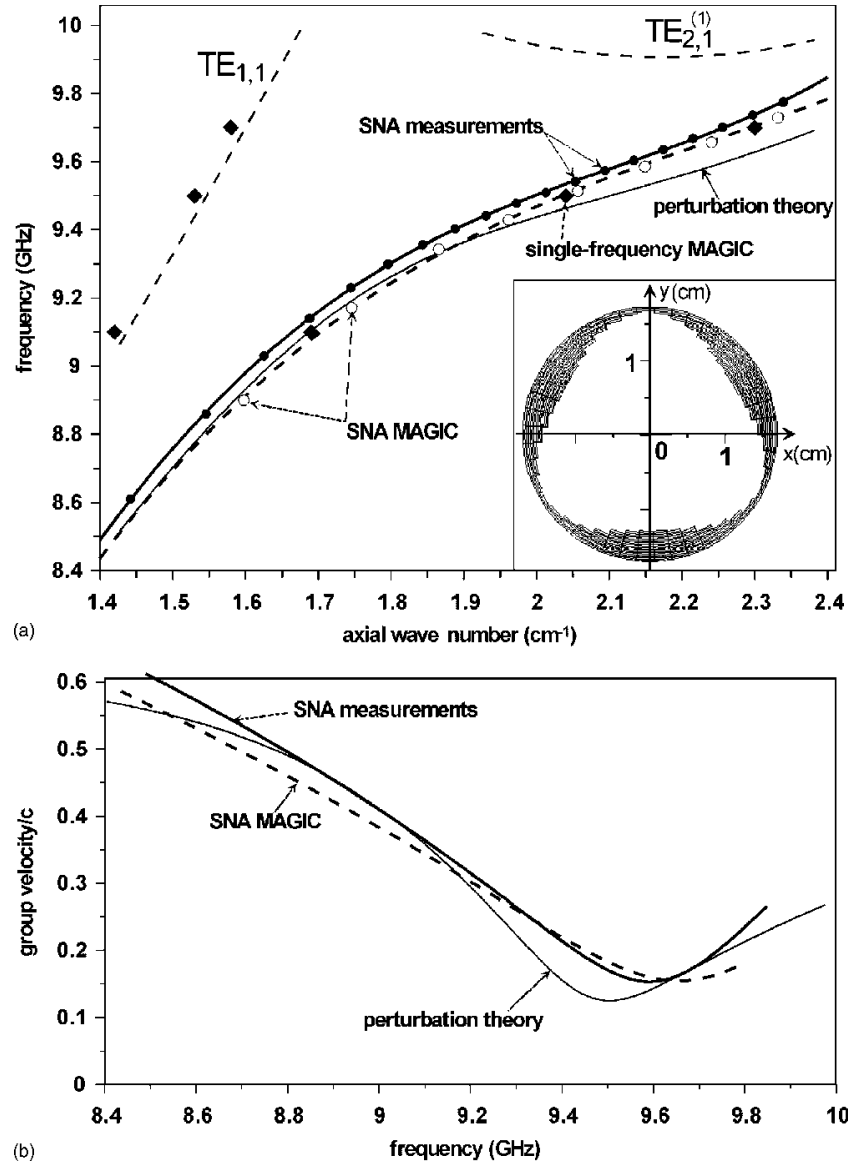

FIG. 6. Dispersion characteristics for compressor waveguide 1 (inset shows MAGIC approximation of the waveguide cross section).

adaptor connected to port 1 and to port 2, respectively, of the VNA. This configuration allowed the reflections to be minimized and the dispersions $k_{z}^{-}(f)$ and $k_{z}^{+}(f)$ to be measured separately by alternating the rotation of the modes via a $90^{\circ}$ turn in the orientation of the polarizers. The VNA was calibrated using a ten-term, LRL calibration that excluded isolation. The calibration planes were each set to be at the connection planes between the elliptical polarizer and the helical taper at each end of the experimental setup. First, the phase difference between the calibration planes as a function of frequency was digitally recorded for the configuration described above. Second, the measurements were repeated with the regular corrugated section removed (see Fig. 8 inset). The measured phases were then digitally processed resulting in continuous functions (without $360^{\circ}$ jumps) which were subtracted from each other. This procedure enabled the impact of only the regular helix to be selected. If at the minimum frequency of the measurements, $f_{\text {min }}$, the condition $k_{z}\left(f_{\text {min }}\right) D>2 \pi$ is satisfied then after processing an uncertainty of $2 \pi n$ (where $n$ is integer) in the phase $\Delta \psi$ still remains, but, at sufficiently short length $D$, it can reliably be deduced by a rough approximation of the dispersion being measured.

\section{3D NUMERICAL SIMULATIONS}

Two different methods were applied to obtain the dispersion characteristics of helically corrugated waveguides
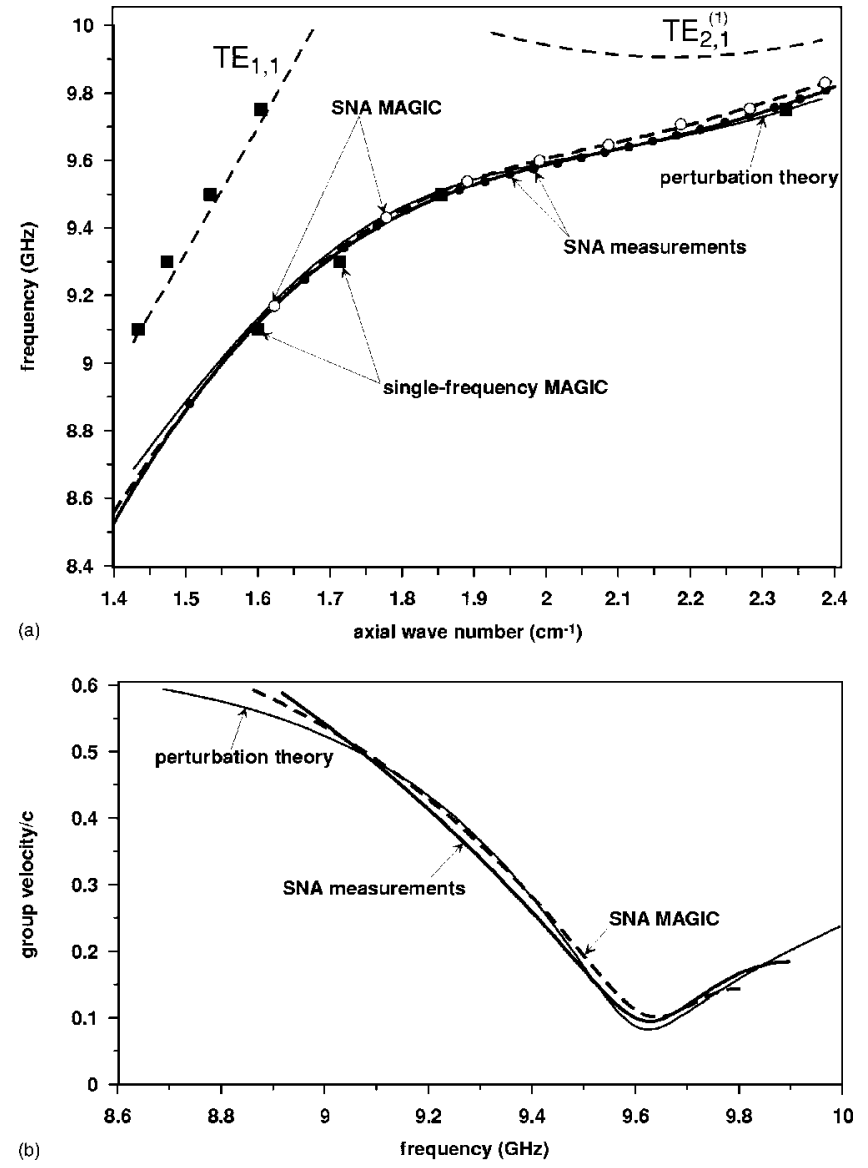

FIG. 7. Dispersion characteristics for compressor waveguide

from simulations with the 3D finite-difference time-domain code MAGIC. The first method, hereinafter referred to as SNA MAGIC, was analogous to that used for the SNA measurements discussed in Sec. IV. Being a time-domain code, MAGIC allows the continuous frequency characteristics of a linear object (like a "cold" hollow structure) to be calculated by performing a temporal Fourier transform of its response to a microwave pulse having a broad frequency bandwidth.

Using this option, a configuration similar to the SNA measurements was simulated, namely, a linearly polarized broadband pulse formed in the $\mathrm{TE}_{1,1}$ mode was injected into a circular waveguide and then propagated through a helical up-taper, regular helix, helical down-taper, and short smoothbore section, at the end of which the electric field was analyzed. The $\mathrm{TE}_{1,1}$ mode was injected and output from the simulation sampled in a waveguide section far above cutoff to ensure that the input and output phase velocities were well matched. In order to simplify the modeling of the helical waveguide surface, a cylindrical coordinate system was used in MAGIC. This excluded from the analysis the electromagnetic fields on the $z$ axis $(r=0)$, and therefore a temporal Fourier transform of the radial electric field at a position slightly shifted from the axis was used as an equivalent to the SNA frequency scan (Fig. 3), which was then processed in the same way to obtain the dispersion diagram.

For a relatively short structure, the exact experimental 

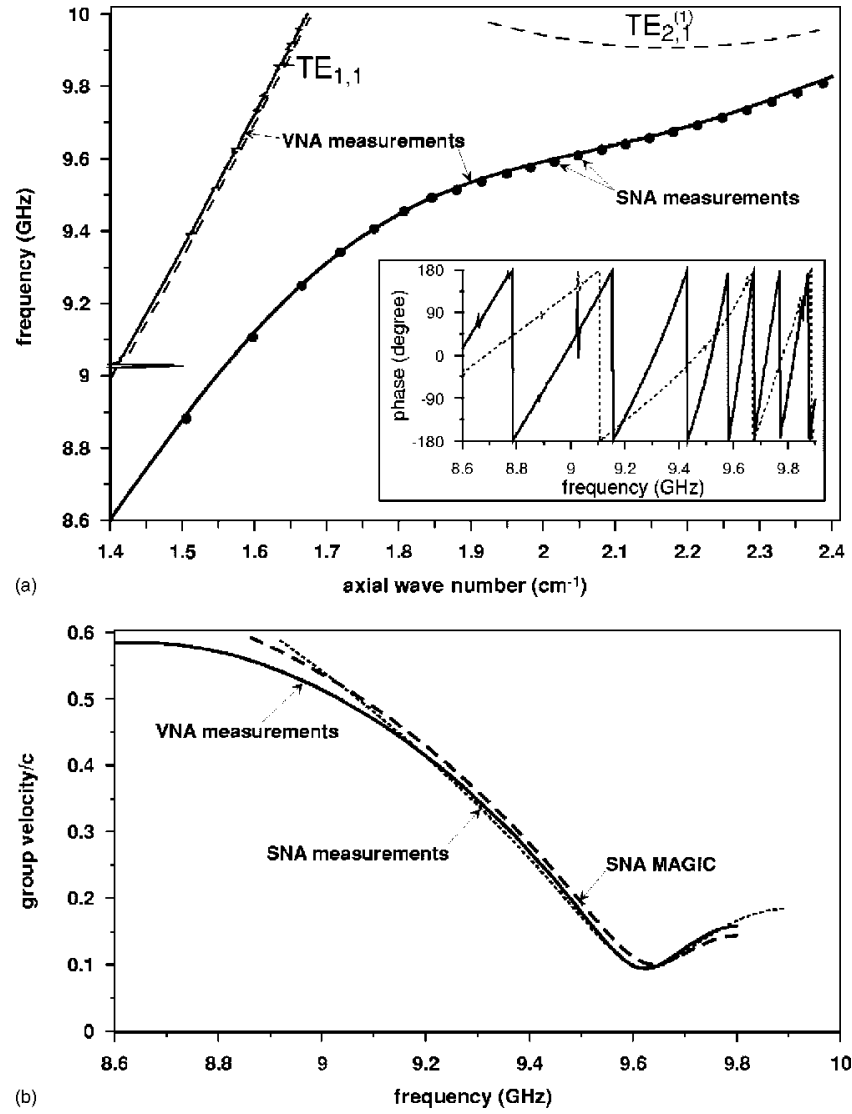

FIG. 8. Dispersion characteristics for compressor waveguide 2 including the VNA measurements [inset shows the data from VNA before processing, including (solid line) and excluding (dashed line) an eight-period helix].

configuration was simulated with MAGIC (Fig. 3) to validate the method and to optimize the size of the numerical cells. The dimensions of the MAGIC cells required to ensure good agreement with results of the measurements were as follows: $d r=l / 7, d \varphi=10^{\circ}, d z=d / 30$ (Fig. 6 inset). A further decrease in all cell dimensions by a factor of 1.5 resulted in a very small shift (less than $0.43 \%$ ) of frequencies of the minima which proved that the numerical simulations had good convergence and reliability. An advantage of this method was that one MAGIC run allowed a wide frequency region to be instantly analyzed, but in this case it was difficult to extract information about the electromagnetic field distribution over the volume (selected plane or line) at a defined frequency.

The second method of simulation, hereinafter referred to as single-frequency MAGIC, set the source of the microwaves at a defined frequency. In this case an eigenwave of a helical waveguide was excited by introducing a circularly polarized $\mathrm{TE}_{1,1}$ mode to the helix through a smooth helical taper similar to that used in the VNA measurements. After a sufficiently long time to ensure that the electromagnetic field was established over all the structure, a snapshot of the radial electric field distribution along a line parallel to the $z$ axis was taken, and its spatial Fourier transform for a region with constant amplitude of the corrugation was performed (Fig. 4). If a left-handed $\mathrm{TE}_{1,1}$ mode was introduced (exciting the eigenwave of main interest) and the line of the $E$ field analysis was sufficiently shifted from the axis, then two clear maxima were seen in the Fourier transform, one of which at smaller axial number corresponded to the spatial harmonic $W_{2}$ (modified $\mathrm{TE}_{2,1}$ mode) while the other (shifted by the Bragg periodicity vector $k_{B}$ ) corresponded to the spatial harmonic $W_{1}$ of the eigenwave. A small admixture of a righthanded $\mathrm{TE}_{1,1}$ mode at the input resulted in the appearance of a maximum at the axial wave number $k_{z}^{+}$of the weakly perturbed wave (Fig. 4). Thus all the axial-wave-number values of interest can be found at a defined frequency. In addition, important information about the field magnitude and its distribution can be obtained using this method. The accuracy of these simulations for determining the axial wave numbers improved with increasing length of the helical structure. In the single-frequency MAGIC simulations performed, structures with an operating length of more than 30 periods were analyzed, which ensured a relative accuracy for the axial wave numbers $k_{z}^{+}$and $k_{z}^{-}$of better than $1 \%$.

\section{RESULTS}

The results of a study of three different structures with three-fold helical corrugations used in $X$-band GTWT and pulse compression experiments are presented and discussed below.

One structure, hereinafter referred to as the GTWT waveguide, had the following parameters: mean radius $r_{0}$ $=1.4 \mathrm{~cm}$, period $d=3.9 \mathrm{~cm}$, corrugation amplitude $l$ $=0.22 \mathrm{~cm}$ (coupling coefficient $\kappa=0.117$ ), length of regularly corrugated section $L_{\text {reg }}=60 \mathrm{~cm}$, and length of each taper $L_{\text {tap }}=12 \mathrm{~cm}$; with the structure able to be disassembled in two halves. In this case the SNA MAGIC simulations of the whole length of the structure agreed very well with the corresponding SNA measurements (Fig. 3). The singlefrequency MAGIC simulations performed for a structure having a $136.5 \mathrm{~cm}(35 d)$ long regularly corrugated section showed a very small difference of $0.26 \%$ (which is less than the accuracy of the method) between the axial number $k_{z}^{+}$of the weakly perturbed mode and that of the unperturbed $\mathrm{TE}_{1,1}$ mode. These simulations resulted also in a difference of less than $1 \%$ for the value of the operating eigenmode axial wave number $k_{z}^{-}$when comparing the results obtained from SNA MAGIC, SNA measurements, and the perturbation theory [Fig. 5(a)]. The difference between the results becomes more evident if the frequency dependences of the group velocity obtained from the different methods are compared. From Sec. I it can be recalled that the eigenwave that has a constant group velocity over the operating frequency band is the most favorable operating mode for a GTWT. Perturbation theory predicts $22 \%$ variation of the eigenmode group velocity for the frequencies between $8 \mathrm{GHz}$ and $10 \mathrm{GHz}$, whereas the SNA MAGIC simulates a smaller variation of $17.5 \%$, which is closer to that obtained from the SNA measurements.

Two other structures used as microwave pulse compressors were analyzed. Both structures had the same mean radus $r_{0}=1.47 \mathrm{~cm}$, period $d=2.89 \mathrm{~cm}$, and length of tapers $L_{\text {tap }}$ $=11.56 \mathrm{~cm}(4 d)$, but different corrugation amplitudes and 
lengths of the regularly corrugated sections: $l=0.175 \mathrm{~cm}(\kappa$ $=0.098), L_{r e g}=184.96 \mathrm{~cm}(64 d)$ for compressor waveguide 1 , which could be disassembled into single-period sections, and $l=0.14 \mathrm{~cm}(\kappa=0.078), L_{\text {reg }}=208.08 \mathrm{~cm}(72 d)$ for compressor waveguide 2 , which could be disassembled into eight period long sections.

In the case of compressor waveguide 1 , the dispersion obtained from the SNA MAGIC technique, simulating a 14period long structure bounded by one-period long tapers, was reasonably close to that obtained from the SNA measurements [Fig. 6(a)]. The results of the single-frequency MAGIC simulations involving a structure with a 35-period long regular section were in very good agreement with the SNA MAGIC method results for the operating eigenmode dispersion and showed again an insignificant difference between dispersions of the nonoperating and unperturbed $\mathrm{TE}_{1,1}$ modes [Fig. 6(a)]. In contrast to the GTWT waveguide, the pulse compression application required the operating group velocity to be significantly changed over the operating frequency region. Moreover, the minimum value of the group velocity was very important for the compressor design (the optimum compressor length is nearly proportional to this value); therefore the difference in the results obtained by perturbation theory and those obtained from the measurements and MAGIC simulations, which is not so evident in the dispersion diagram [Fig. 6(a)], becomes essential if one considers a group velocity vs frequency function [Fig. 6(b)]. The minimum value predicted by the perturbation theory was $\nu_{g r(\min )}=0.125 c$, whereas the MAGIC simulations resulting in $\nu_{g r(\min )}=0.155 c$ gave a much better agreement with measurements $\left(\nu_{g r(\min )}=0.153 c\right)$.

In the case of compressor waveguide 2 , for which the best results were achieved in the experiment on pulse compression, all the methods to obtain the eigenwave dispersion characteristics discussed above were applied. Structures having lengths of regularly corrugated sections equal to 12 periods and 35 periods, each bounded by one-period tapers, were used in SNA MAGIC and single-frequency MAGIC simulations, respectively (Fig. 7). The whole length of the structure was used for the SNA measurements whereas an eightperiod long section of regular corrugation bounded by the tapers was measured with the VNA (Fig. 8). In contrast to the results for compressor waveguide 1 , in the case of compressor waveguide 2 having essentially a shallower corrugation, all the methods including perturbation theory resulted in practically the same results, which confirmed the reliability of the MAGIC simulations and indicated the region of parameters (coupling coefficient $\kappa=0.078$ ) where the simple analytical approach can give sufficiently high accuracy.

\section{CONCLUSION}

Several of the theoretical and experimental methods discussed are generally applicable for a wide region of parameters and can be used for accurate analysis of the dispersive properties and design of helically corrugated waveguides. A simple theoretical approach based on the method of perturbation can be used for a preliminary choice of the helical structure and is more accurate for a relatively small corrugation amplitude $(\kappa \leqslant 0.1-0.15)$. Depending on the required dispersion properties, perturbation theory provides reasonable or sufficient accuracy. The SNA MAGIC simulations have the advantage of being able to generate sufficient data for the dispersion using comparatively limited computer capabilities and a short run time, but more importantly can be used for increased accuracy in the analysis of a specific eigenwave dispersion characteristic. The single-frequency MAGIC simulations allow an estimation of the systematic inaccuracy and validation of the results obtained from SNA MAGIC. Moreover, these simulations enable detailed analysis of the electromagnetic field inside the structure to be carried out. The VNA measurements involving a relatively short piece of the helical waveguide allow the dispersion characteristics to be accurately found before the full-length helix is constructed, whereas the SNA measurements which rely on more readily available equipment can be effectively used for a final "cold" test of a manufactured structure.

\section{ACKNOWLEDGMENTS}

This work was supported by the EPSRC U.K., the University of Strathclyde, the Presidium of the Russian Academy of Sciences, and the Russian Foundation for Basic Research (Grant No. 04-02-16698). The Anritsu Philanthropy Scheme is gratefully acknowledged for its assistance in providing the vector network analyzer used in the experiments. The authors would like to thank Jamal Hamid, Ken Foan, and Paul Holes of Anritsu for useful discussions. The assistance of Lars Ludeking of ATK mission research with the setting up of MAGIC is gratefully acknowledged.
[1] E. A. Abubakirov et al., IEEE Trans. Plasma Sci. 30, 1041 (2002).

[2] M. I. Fuks and N. F. Kovalev, IEEE Trans. Plasma Sci. 30, 1147 (2002).

[3] M. K. Thumm and W. Kasparek, IEEE Trans. Plasma Sci. 30, 755 (2002).

[4] V. L. Bratman et al., Phys. Rev. Lett. 84, 2746 (2000).

[5] V. L. Bratman et al., in Proceedings of the 5th International Workshop on Strong Microwaves in Plasmas, Nizhny
Novgorod, Russia, 2002, edited by A. G. Litvak (Institute of Applied Physics, Russian Academy of Sciences, Nizhny Novgorod, 2003), p. 46.

[6] S. V. Samsonov et al., High Power Microwave Generation, special issue of IEEE Trans. Plasma Sci. 32, 884 (2004).

[7] A. W. Cross et al. (unpublished).

[8] S. V. Samsonov et al., Phys. Rev. Lett. 92, 118301 (2004).

[9] N. F. Kovalev, I. M. Orlova, and M. I. Petelin, Radiophys. Quantum Electron. 11, 783 (1968) [Radiophys. Quantum Elec- 
tron. 11, 783 (1968)] .

[10] S. J. Cooke and G. G. Denisov, IEEE Trans. Plasma Sci. 26, 519 (1998).

[11] L. Ludeking, D. Smithe, and T. Gray, Computer code MAGIC, Mission Research Corporation, VA, 2003.
[12] G. G. Denisov and M. G. Reznikov, Radiophys. Quantum Electron. 25, 562 (1982) [Radiophys. Quantum Electron. 25, 562 (1982)] .

[13] G. G. Denisov, V. L. Bratman, A. D. R. Phelps, and S. V. Samsonov, IEEE Trans. Plasma Sci. 26, 508 (1998). 